SUBJECT AREAS:

COMPUTATIONAL

MODELS

COMMUNITIES

Received

15 May 2014

Accepted

22 August 2014

Published

18 September 2014

Correspondence and requests for materials should be addressed to R.D.W. (rowelch@syr.

edu)

\footnotetext{
* These authors contributed equally to this work.

$\uparrow$ Current address: Genzyme Corporation.

$\$$ Current address: SUNY Stony Brook, Department of Biology.
}

\title{
Aggregation Using Ostwald Ripening Equations for Thin Liquid Films
}

\author{
Fatmagül Bahar ${ }^{1 *}$, Philip C. Pratt-Szeliga ${ }^{2 *}$, Stuart Angus $^{1} \dagger$, Jiaye Guo ${ }^{1}+\&$ Roy D. Welch ${ }^{1}$
}

${ }^{1}$ Syracuse University, Department of Biology, ${ }^{2}$ Syracuse University, Department of Electrical Engineering and Computer Science.

When starved, a swarm of millions of Myxococcus xanthus cells coordinate their movement from outward swarming to inward coalescence. The cells then execute a synchronous program of multicellular development, arranging themselves into dome shaped aggregates. Over the course of development, about half of the initial aggregates disappear, while others persist and mature into fruiting bodies. This work seeks to develop a quantitative model for aggregation that accurately simulates which will disappear and which will persist. We analyzed time-lapse movies of $M$. xanthus development, modeled aggregation using the equations that describe Ostwald ripening of droplets in thin liquid films, and predicted the disappearance and persistence of aggregates with an average accuracy of $85 \%$. We then experimentally validated a prediction that is fundamental to this model by tracking individual fluorescent cells as they moved between aggregates and demonstrating that cell movement towards and away from aggregates correlates with aggregate disappearance. Describing development through this model may limit the number and type of molecular genetic signals needed to complete $M$. xanthus development, and it provides numerous additional testable predictions.

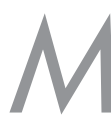

yxococcus xanthusis a flexible rod-shaped bacterium that can move across a semi-solid surface in either direction along its long axis ${ }^{1}$. Under laboratory conditions, $M$. xanthus is grown either vegetatively in liquid culture or as a motile biofilm, called a swarm, on agar. A small swarm can be initiated by spotting a few microliters of liquid culture on an agar surface and letting it dry. If the agar is nutrient-rich, the swarm will expand outward in all directions across the surface through multicellular projections called flares ${ }^{2}$. If the agar is non-nutritive, movement turns inward and the swarm appears to contract. Within several hours, the millions of starving cells self-organize into several hundred dome shaped aggregates, each of which contains many thousands of cells. Following this period of aggregation, a subset of cells at the interior of each aggregate differentiates to become quiescent spores. When aggregation and sporulation are both completed, aggregates are considered to have matured into fruiting bodies. The entire developmental process can take less than 24 hours $^{3,4}$.

Prior research ${ }^{2}$ has reported that only about half the aggregates that appear at the beginning of the aggregation process will persist through maturation to become fruiting bodies, while the other half will shrink and disappear. Xie $e a^{5}$ sought to identify differences between persisting and disappearing aggregates, and found that aggregate size was the only statistically reliable determinant: persisting aggregates were larger. This determinant can be used to accurately predict disappearing and persisting aggregates, but it provides no model to explain why or how this happens.

At present, the only models for M. xanthus aggregation are based on the "traffic jam" hypothesis, which states that during aggregation cells will clump together and become stuck, or jammed, at positions within the swarm when the concentration of cells is sufficiently high. Cells within these traffic jams undergo a transition from motile to non-motile, and in this way an aggregate is initiated. Additional motile cells encounter these initial aggregates, and they become jammed and undergo the same transition. Variations on this model based on a "capitalistic economic system" or reaction diffusion mechanism have been proposed for Pseudomonas aeruginosa ${ }^{6}$ and Dictyostelium discoideum ${ }^{7}$ respectively. The problem with these types of models in M. xanthus is incorporating a method for disappearance. Agent based models implementing the traffic jam hypothesis accurately predict aggregate formation, but have no disappearance component ${ }^{8-12}$.

In this report we propose a new hypothesis for controlling aggregate disappearance based on a model of Ostwald ripening in thin liquid films $\mathrm{s}^{13}$. We implement previously published two-dimensional pairwise equations that describe Ostwald ripening in a simulator (o-simulator) that operates over an area large enough to cover 

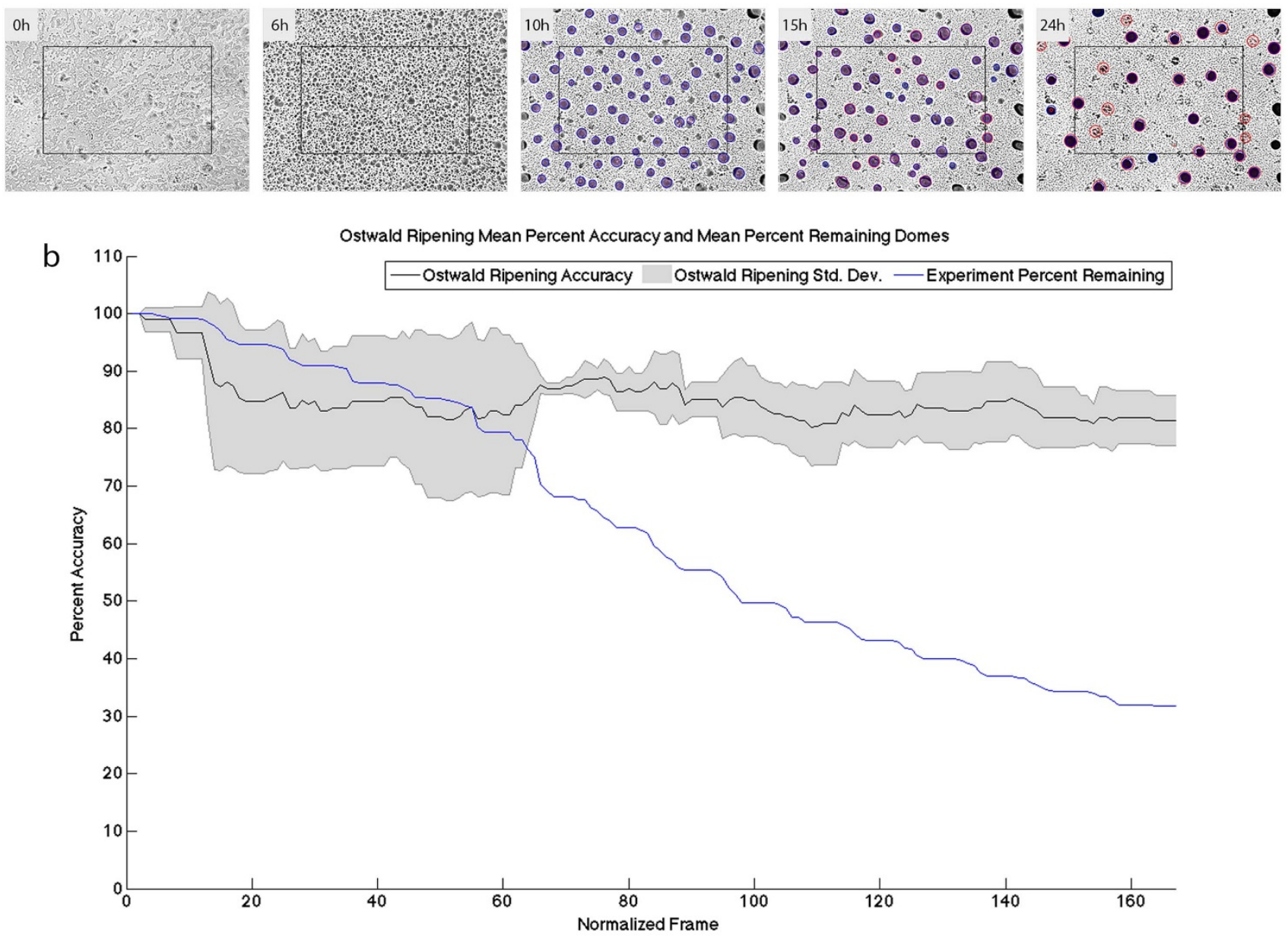

Figure $1 \mid$ M. xanthus aggregation and accuracy of the o-simulation. (a) Five microcinematography images selected from a time-lapse image stack that shows the progression of aggregation (taken from stack 19). Elapsed time is indicated in the upper left corner of each frame (0,6, 10, 15 and $24 \mathrm{~h}$ ). Blue circles with ID numbers represent the positions and volumes of aggregates, and red circles represent the positions and volumes of simulated aggregates (10, 15 and $24 \mathrm{~h}$ ). Blue circles are not visible in frame 3, which is the first e-frame, because red circles exactly overlap blue circles on that frame. (b) The black line displays the average accuracy $( \pm 1 \mathrm{SD})$ of the disappearance model over 5 prediction replicates. The blue line represents the average percent aggregates remaining for the 20 replicates.

dozens of aggregates. This o-simulator predicts the change in volume of each aggregate based on its current volume and the volume and relative proximity of its neighbors. We then test our hypothesis by experimentally deconstructing $M$. xanthus development, isolating the disappearance of aggregates, observing the transient swarm structures and the movements of individual cells, and then matching these observations to the results produced by the o-simulator.

A method similar to this was successfully applied in M. xanthus to provide a mechanism for describing the density waves, called ripples $^{14-16}$, that sometimes travel across the surface of a swarm. Welch et $a l^{14}$ were able to partially reveal the mechanism of rippling by isolating one set of opposing ripples, observing their behavior and the behavior of individual cells within ripples, and then matching these observations with a set of equations that describe non-zhabotinsky waves.

\section{Results}

A quantitative comparison between Ostwald ripening and $M$. xanthus aggregation. To observe and record swarm-scale dynamics of $M$. xanthus aggregation, we used bright field time-lapse microcinematography at $60 \times$ magnification, 1 frame $/ \mathrm{min}$, covering an $\sim 50 \mathrm{~mm}^{2}$ area from an interior section of a $1 \mathrm{~cm}$ diameter swarm composed of $2.5 \times 10^{7}$ wild-type (DK1622) cells on starvation TPM agar (Figure 1a). A stack (movie) of 1440 sequential images (frames) were taken for each sample over of 24 hours, beginning no later than $15 \mathrm{~min}$ after starvation onset (for a detailed protocol, see methods). We independently repeated this procedure 20 times to produce 20 replicate experiments.

Individual cells are not distinguishable under these conditions, but we can estimate from the cell concentration of the liquid culture, the volume spotted onto agar, the area of the initial swarm following liquid absorption and evaporation, and the area of the microscope field-of-view, that there are approximately $8.3 \times 10^{6}$ cells within the field-of-view at starvation onset (Figure 1a, Frame 1). Images recorded at starvation onset have a dappled appearance, with irregular variations in grayscale representing local differences in cell density. Initial aggregates begin to emerge as dark spots within the first 6 to 8 hours (Figure 1a, compare frames 1 and 2). We define each microcinematography experiment frame (e-frame) as a data structure that contains the positions and radii of each aggregate in the frame. We begin the analysis of aggregate disappearance at the first eframe (Figure 1a, frame 3), when initial aggregates are clearly visible and distinguishable from earlier and more transient fluctuations in grayscale.

To create the first e-frame, the position and size of each aggregate is manually determined, recorded, and then tracked for the rest of the image stack using a semi-automated method. Briefly, our method is to manually curate changes in the size and position of all aggregates 
every tenth image, record these changes and interpolate them over the intervening nine sequential images, manually check and adjust the interpolation and move on to the next set of ten images. Using this method we tracked changes in the position and size of a total of 1,727 aggregates. Tracking data is visualized in Figure 1a as blue circles in frames 3,4 , and 5 , and in each of the movies provided in supplementary materials.

To synchronize the start of data collection and the start of the osimulator, the size and location of aggregates in the first e-frame were used to construct the first o-simulator frame (o-frame). These data are visualized in Figure 1a as red circles in Figure 1a frames 3, 4, and 5 , and in each of the movies provided in supplementary materials. Because Figure 1a frame 3 represents both the first e-frame and first o-frame, the red circles and blue circles are identical and exactly overlap. The blue circles are rendered behind the red circles in the image, and so only the red circles are visible.

The simulation examines each pairwise set of circles in each oframe, and adjusts their size according to the following equations

$$
L^{k}=\left|X_{1}^{k}-X_{2}^{k}\right|
$$

$$
\begin{gathered}
\Delta V=\tau \frac{4 \pi^{4 / 3}}{\log \left(L^{k} /\left(V_{1}^{k}\right)^{1 / 3}\right)+\log \left(L^{k} /\left(V_{2}^{k}\right)^{1 / 3}\right)}\left(\left(V_{1}^{k}\right)^{-1 / 3}-\left(V_{2}^{k}\right)^{-1 / 3}\right) \\
V_{1}^{k+1}=V_{1}^{k}-\Delta V \\
V_{2}^{k+1}=V_{2}^{k}-\left(V_{1}^{k+1}-V_{1}^{k}\right)
\end{gathered}
$$

where $\mathrm{X}_{1}{ }^{\mathrm{k}}, \mathrm{X}_{2}{ }^{\mathrm{k}}, \mathrm{V}_{1}{ }^{\mathrm{k}}$ and $\mathrm{V}_{2}{ }^{\mathrm{k}}$ represents the positions and volumes at time $\mathrm{k}$ and $\mathrm{V}_{1}{ }^{\mathrm{k}+1}$ and $\mathrm{V}_{2}{ }^{\mathrm{k}+1}$ represents the volumes at time $\mathrm{k}+1$. The outcome from these pairwise equations is that the larger aggregate will increase in volume at the expense of the smaller aggregate, and the amount gained by the larger aggregate will be equal to the amount lost by the smaller aggregate. Distance between aggregates mediates the volume change, so that closer aggregates have more effect than distant aggregates. An aggregate will disappear when the volume drops below a minimum threshold, and touching aggregates will merge into a single aggregate of their combined volume. For a detailed description of the o-simulation, see methods.

To measure the accuracy with which the o-simulation can predict the timing of aggregate disappearance, we first determined the appropriate rate of mass transfer between droplets, which is represented as tau $(\tau)$ in the volume equation (Eq. 2 ). The rate of mass transfer between cells appears to undergo a synchronous increase midway through development, and so $\tau$ was determined twice $(\tau-1$ and $\tau-2) ; \tau-1$ was determined for the first half of the o-simulation, and $\tau-2$ was determined for the second half (see methods). To calculate these values, we optimized $\tau$ for both halves using 15 randomly chosen replicates as training replicates, resulting in a $\tau-1$ of 160 and a $\tau-2$ of 700 . We then determined the accuracy for the remaining 5 prediction replicates. Specifically, we paired every aggregate to its corresponding o-simulated aggregate from each sequential e-frame and o-frame, and then employed a scoring mechanism that measured how accurately the o-simulation matches experiment with respect to aggregate disappearance. The scoring starts at $100 \%$ accuracy because the first e-frames and first o-frames are identical. Sequential e-frames and o-frames were then examined, and if one half of an "e-frame::o-frame" aggregate pair disappeared while the other half persisted, the accuracy score would decrease accordingly for that frame and subsequent frames. If the other half of the pair disappeared in a later frame, the accuracy score for that pair would be restored for the remainder of frames. In this way the score represents the accuracy for each frame, so that changes in accuracy can be plotted as a function of time (Figure $1 \mathrm{~b}$ ).

Aggregate disappearance occurred during the entire period covered by the o-simulation, as seen in Figure $1 \mathrm{~b}$ (blue line). The accuracy score initially fell to $\sim 80 \%$ after the first 20 frames, remained relatively constant until 60 frames, and then increased slightly, reaching a final score of $81 \% \pm 4.29 \% \mathrm{SD}$. The overall average accuracy score was $85.1 \% \pm 7.09 \%$ SD during the entire 10 to 14 simulated hours since the first e-frame. The relative consistency of the accuracy score indicates that the o-simulation was also able to match the timing of disappearance reasonably well.

To compare our results to previous research, we repeated this comparison using the only other proposed determinant to predict aggregate disappearance in $M$. xanthus, the size threshold hypothesis proposed by Xie et al ${ }^{5}$. The size threshold hypothesis does not include a time component; small aggregates below a threshold simply disappear while the larger ones above the threshold persist. From this hypothesis we created a size simulation (s-simulation) that predicts aggregate disappearance if it is below a specified threshold size based on the first size frame (s-frame), which is also matched to the first e-frame. We determined the best size threshold for the same 15 training replicates that we used for the o-simulation, executed the $\mathrm{s}$-simulation in same manner as the o-simulation, and then generated an accuracy score for the 5 prediction replicates. Because the size threshold hypothesis includes no time component, the rate of mass transfer $(\tau)$ is not a component of the s-simulation.

We compared the accuracy scores of the s- and o-simulations with respect to time (Figure 2). The mean accuracy score of the o-simulation is always greater than $80 \%$. In contrast, the mean accuracy score of the s-simulation is less than $40 \%$ after the first 20 frames, but this is due to the lack of a time component in the size simulation; between the first and second s-frames, everything below the threshold disappears, and all sequential s-frames are identical from the second to the last frame. In other words, the second s-frame is the last s-frame, and if the s-simulation is an accurate predictor of which aggregates will disappear during development, then s-frames and eframes should converge from the second to the last frames. We observed this effect; the mean accuracy score of the s-simulation increases steadily over the next 60 frames, reaching the average accuracy of the o-simulation by frame 100 . After that, the accuracy of the two simulations remains similar. Therefore we conclude that the Ostwald ripening hypothesis matches the accuracy of the size threshold hypothesis in predicting which aggregates will disappear and, because it includes a mechanism, it is also able to predict when they will disappear.

Experimental validation of Ostwald ripening as the mechanism underlying aggregate disappearance. Ostwald ripening in a thin liquid film represents entropy minimization through the reduction in surface area. The surface area of a hemispherical droplet increases more slowly than its volume, and so the total surface area of the thin liquid film decreases as droplets become larger in size and fewer in number. Ostwald ripening also represents a zero-sum game, so shrinking droplets lose liquid to growing droplets ${ }^{13}$. By analogy, in a developing $M$. xanthus swarm, shrinking aggregates must lose cells to growing aggregates. If aggregates shrink for a different reason, such as cell death, then the aggregation-as-Ostwald ripening analogy does not hold.

To test the analogy, we repeated the development microcinematography experiments using a $1: 150$ mixture of fluorescent $M$. xanthus cells. First we used bright-field microcinematography at $60 \times$ magnification to identify aggregates as either growing or shrinking, then we increased magnification to $150 \times$, positioned the aggregate in the middle of the field-of-view, and used fluorescence microcinematography to track the movement of cells immediately outside the aggregate over 15 minute intervals.

To determine if the net migration of cells was toward or away from an aggregate, we examined the paths of fluorescent cells relative to the centered aggregate; if a cell moved closer to the aggregate, then the cell was designated "towards", if it moved farther from the 


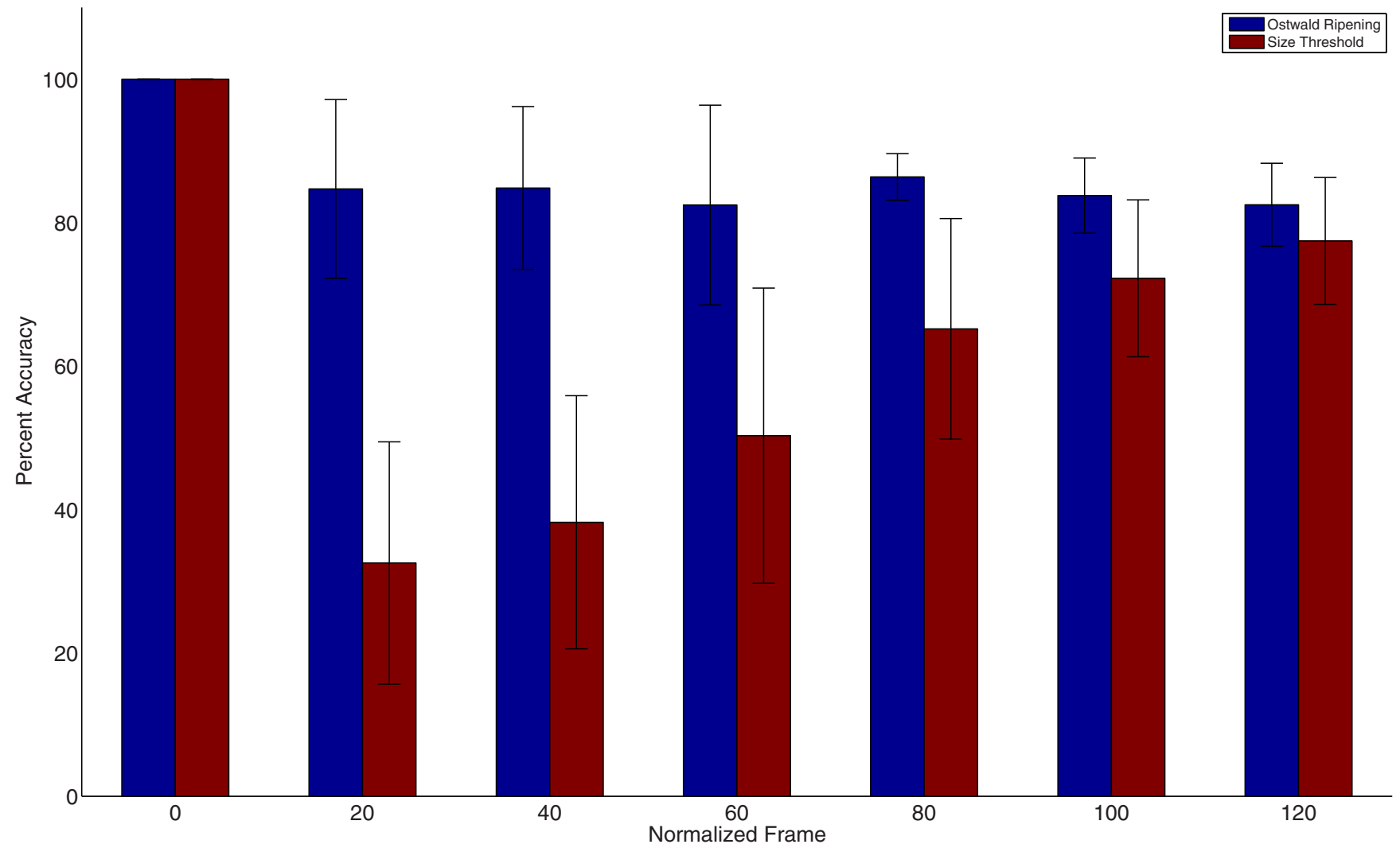

Figure $2 \mid$ A comparison of the average accuracy score between the o-simulator (blue bars) and s-simulator (red bars) with respect to predicting aggregate disappearance over time for 5 prediction replicates. Error bars report one standard deviation.

aggregate it was designated "away", and if there was no net movement it was designated "none". A total of 197 cells were tracked for 12 aggregates (7 growing and 5 shrinking) (Figure 3).

The results are unambiguous. Cells near a growing aggregate (Fig. 3a) are more likely to be moving toward it (Fig. 3c), and cells near a shrinking aggregate (Fig. 3b) are more likely to be moving away from it (Fig. 3d). The overall difference is two-fold (Fig. 3e), which strongly supports the idea that disappearing aggregates shrink because they are losing cells to growing aggregates that persist. Therefore, these data support the aggregation-as-Ostwald ripening analogy.

Characterizing phenotypic boundaries for an aggregation-asOstwald ripening analogy. Ostwald ripening is only one of two behaviors that describe the dynamics of entropy minimization in a thin liquid film. The second behavior is called migration, which describes how droplets move across a thin liquid film. Ostwald ripening and migration can be represented by separate sets of equations, but they are mechanistically linked, and collectively they are called coarsening.

Migration is caused by asymmetries in the density of particles around a droplet as a function of Ostwald ripening by neighboring droplets. $M$. xanthus aggregates sometimes move across the swarm in a manner that appears similar to migration, but the speed and direction of aggregate movement is highly variable. Equations that describe droplet migration on thin liquid films fail to predict aggregate movement any better than equations that describe random movement (supplementary materials).

We note two phenotypic features clearly visible in image stacks from the 20 replicates that may partly explain why aggregate movement is not comparable to droplet migration. We refer to these two features as "snakes" and "scars". Neither snakes nor scars are pre- dicted by a coarsening model, and they may indicate important ways in which coarsening and aggregation are dissimilar. We discuss snakes and scars separately.

Snakes sometimes occur when an aggregate disappears (Figure $4 \mathrm{a}$, also stack 20 inset within frames 800-1000). They can be observed in the image stacks as elongated slightly darker regions that travel quickly away from the disappearing aggregate, usually in two opposing directions. Often a snake will seem to travel directly toward a nearby aggregate. We hypothesize that snakes represent the transfer of large quantities of cells from one aggregate to another through structures called slime trails. M. xanthus cells deposit trails of a polysaccharide matrix, called slime, as they move across agar, and cells encountering previously deposited slime trails tend to follow them ${ }^{17}$. This effect of slime trails functions together with other forces, such as surface tension, to cause the formation of parallel lines of cells, called streams ${ }^{18}$. Our observation that snakes frequently travel in opposing directions may indicate where slime trails have formed, and our observation that snakes frequently travel to nearby aggregates may indicate that slime trails cause a preferential transfer of cells between aggregate pairs.

Scars also sometimes occur when an aggregate disappears (Figure $4 \mathrm{~b}$, also stack 17 inset within frames 1180-1440). Scars seem residual, as though the disappeared aggregate had caused a change in the substrate that permanently altered the structure of the swarm in that region. Cell movement is observed within and around scars, but the scars do not move across a swarm. The overwhelming majority of disappearing aggregates that leave scars also do not move, but even in the few cases where the disappearing aggregates do move, the scars they leave behind do not. We hypothesize that scars represent the aborted maturation of an aggregate to a fruiting body. Perhaps they are the beginnings of mucoid stalks that would have suspended the bolus of spores. 

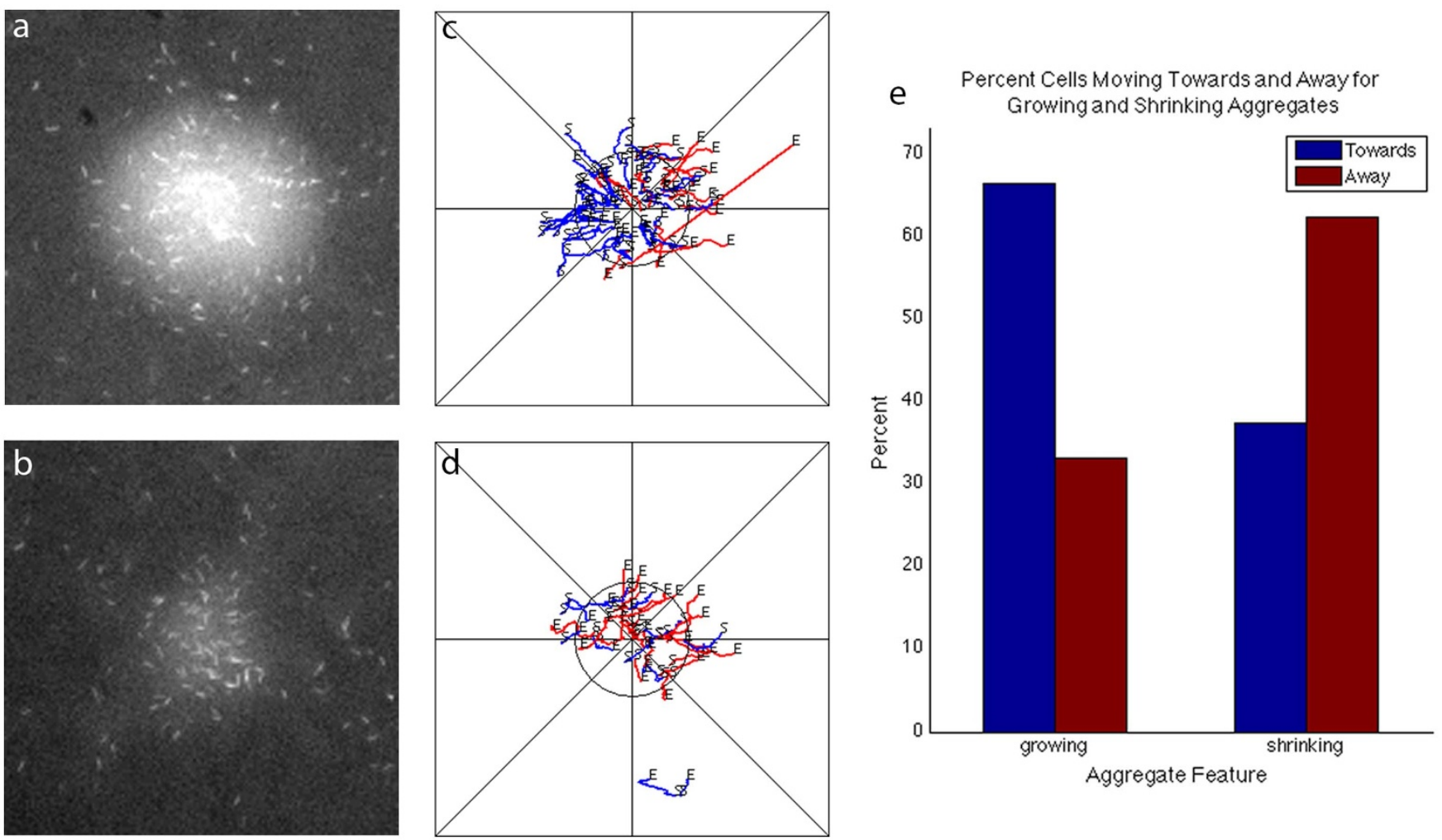

Figure 3 Towards/away comparison of fluorescent cells moving in and around growing and shrinking aggregates. (a) Fluorescent images of growing aggregates and (b) shrinking aggregates with fluorescent cells visible in and around them. (c) Total individual tracked cells on seven growing and (d) five shrinking aggregates. Blue lines represent cell tracks moving toward the aggregates, and red lines represent cell tracks moving away from the aggregates. The letters 'E' within the tracks indicates the endpoints of tracks, and the letters ' $S$ ' indicates the starting points of tracks. (e) The total number of cells tracked as moving 'towards' (blue bars) or 'away' (red bars) from growing and shrinking aggregates.

We further hypothesize that snakes and scars are phenotypic features that indicate two aspects of aggregation that make it unlike coarsening. Snakes indicate that cells leaving a disappearing aggregate do not diffuse evenly into a swarm, but preferentially follow slime trails. This would cause coarsening and aggregation to deviate with respect to both movement and disappearance, since the asymmetry of cell density would be greatly increased, and cell transfer between pairs of aggregates would depend on more than size and proximity. Scars indicate that aggregate movement may be disconnected from disappearance. In coarsening, Ostwald ripening and migration are a

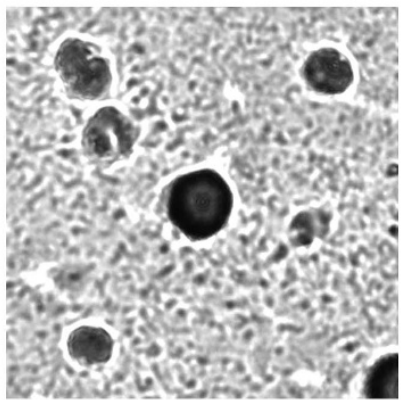

b

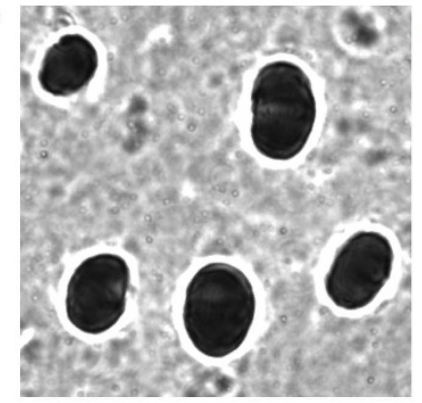

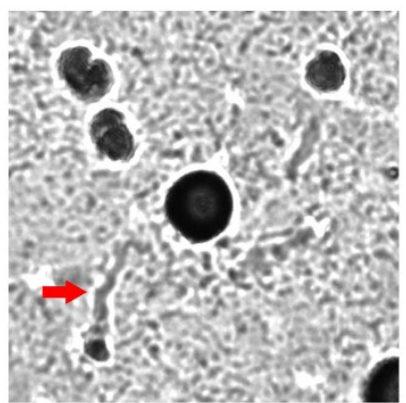

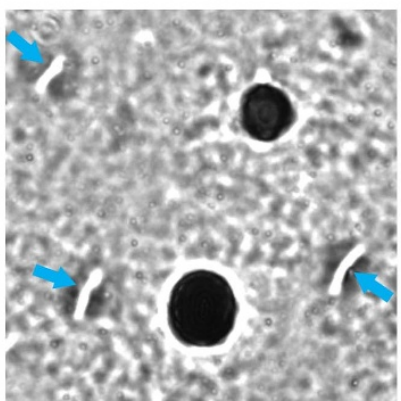

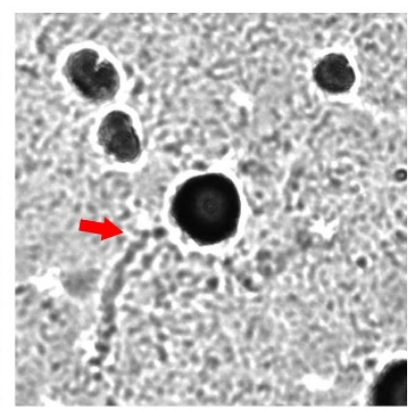

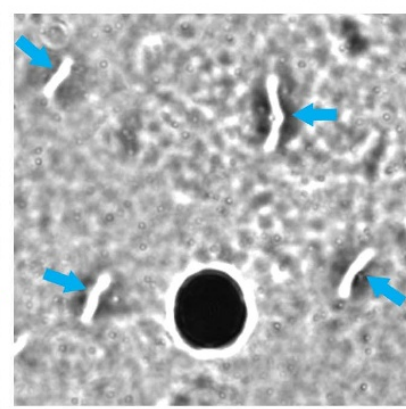

Figure $4 \mid$ Representative examples of snakes and scars. (a) A snake (red arrow) traveling from a disappearing aggregate to a growing aggregate (stack 20). (b) Scars remaining (blue arrows) where aggregates have disappeared (stack 17). 
linked by the same underlying mechanism. In contrast, if aggregates can anchor themselves to the substrate so that they don't move, but they still exchange cells and may disappear, then aggregate movement and disappearance have been mechanistically uncoupled. Perhaps snakes and scars are some visible manifestations of why our predictions for movement are not accurate, and why the accuracy of the o-simulations top out at $\sim 85 \%$.

\section{Discussion}

We have demonstrated that a model simulating $M$. xanthus aggregation based on equations that describe Ostwald ripening in thin liquid films is able to predict which aggregates will persist and which will disappear with $\sim 85 \%$ accuracy. We also tested and validated one of the central predictions of such a model: that aggregates grow because of cell accumulation, and shrink and disappear because of cell loss. This provides a mechanism for the size threshold determinant ${ }^{5}$, and is the first alternative to the traffic jam model that includes aggregate disappearance.

Our argument from analogy is not to claim that $M$. xanthus aggregation is coarsening in thin liquid films; enough is known about $M$. xanthus genetics, cellular behavior, and multicellular self-organization to refrain from making this much stronger claim. For example, we know that $M$. xanthus synchrony and symmetry breaking is under genetic control by molecular signals and pathways that coordinate swarm cell movement ${ }^{2,19}$. We also know that $M$. xanthus cells are active ${ }^{1,20,21}$, they move along a semi-rigid axis with a genetically controlled reversal frequency ${ }^{22,23}$, and they form streams that steer them over long distances ${ }^{18,24}$. Finally, we know that the shape and structure of an M. xanthus fruiting body requires the coordination of thousands of cells because it is much more than a droplet, with a cell-free mucoid stalk and a bolus of myxospores at the center ${ }^{25}$.

Instead, our argument is that Ostwald ripening is similar enough to $M$. xanthus aggregation that it can provide testable genetic and behavioral hypotheses and a mechanistic model consistent with Occam's Razor. An Ostwald ripening aggregation model reduces the number of developmental decisions that must be assigned to quorum sensing and cell-contact mediated mechanisms. It also provides a hypothesis that links individual cell movements to an important aspect of swarm self-organization.

\section{Methods}

Growth and development culture conditions. Liquid cultures of $M$. xanthus were grown in agitating nutrient CTTYE (1.0\% Casitone [Difco], $0.5 \%$ yeast extract [Difco], 10.0 mM Tris- $\mathrm{HCl}$ [pH 8.0], $1.0 \mathrm{mM} \mathrm{KH2PO} 4$, and $8.0 \mathrm{mM} \mathrm{MgSO} 4)$, and development assays were performed using starvation TPM agar $(10.0 \mathrm{mM}$ Tris- $\mathrm{HCl}$ (pH 7.6), 1.0 mM KH2PO4, $8.0 \mathrm{mM} \mathrm{MgSO} 4$, and 1\% agar). To prepare cells for development assays, growing cells were harvested from liquid culture at Klett 80-120 (log phase). Development assays were prepared as previously described ${ }^{2,22}$ with the following modifications: The gasket used to contain the agar was thicker $(2.5 \mathrm{~mm})$ than the gasket used to provide the airspace $(0.5 \mathrm{~mm})$; the initial spot had a concentration of $5.0 \times 10^{9}$ cells $/ \mathrm{mL}$. Microcinematography was performed at $30^{\circ} \mathrm{C}$. Wild-type DK1622 was used for development assays to record the behavior of aggregates, and a 1/150 mixture of DK1622 and fluorescent DK10547 $7^{14}$ was used to record the behavior of individual cells.

Time lapse microcinematography and image processing. For aggregates: To obtain the data for aggregates, we created a custom C\# application that allows identification to be performed semi-automatically. First, the image within the stack containing the highest number of aggregates is selected as a starting point (this is usually within 10 hours after the initiation of development). The user of the application clicks on the image and a blue circle appears that can be moved by dragging or expanded/reduced in size by moving the mouse wheel. Once a frame is completely annotated, the designated aggregates (blue circles) in the frame are extended ten frames forward. The tenth frame is adjusted for changes by the user, and then the positions and radii are interpolated within the ten frame range by the application. This process is repeated for the entire movie in ten frame batches. The positions and radii of each annotated aggregate in each frame are saved to a file format that can be read using the simulations written in Java.

For tracking individual cells: To obtain the data on the movement of individual cells, we performed microcinematography on developmental cultures using a 150:1 mixture of wild-type and GFP-expressing cells that develop as wild-type. At the period of development when disappearance is occurring, we identified individual aggregates as either growing or shrinking, increased magnification to $150 \times$, moved the slide so that the aggregate was in the middle of the field-of-view, and then continued, acquiring time-lapse fluorescence images every minute. Using these images we were able to identify and track individual cells moving around a growing or shrinking aggregate.

We manually recorded $\mathrm{x} / \mathrm{y}$ coordinates of the cell at each time step and placed the coordinates into an excel spreadsheet. A Java program parsed the excel spreadsheet and visualized each trail relative to the center of the aggregate on an image. Then we manually determined whether each trail was moving towards, away or none, and inserted this new information into an updated spreadsheet. Another Java program then read the updated spreadsheet, colored the annotated trails, and placed them on composite images (Figure $3 \mathrm{c}$ and $3 \mathrm{~d}$ ). We were then able to calculate the percentage moving towards/away when the aggregates were growing and shrinking (Figure 3e).

Dynamical model to predict aggregate behavior. The o-simulator has a front end that can read the data files from the custom C\# application which contain aggregate position and radii (first e-frame). The front end creates unique identifiers for each aggregate in the first e-frame and matches the closest aggregate in subsequent eframes to associate aggregates by identifier throughout the image stack.

We seed the first $\mathrm{o}$-frame of the $\mathrm{o}$-simulation with the position and radii data from first e-frames as the only input (besides the free variables $\tau-1$ and $\tau-2$ ). For each subsequent $\mathrm{o}$-frame, the discretized pairwise equations for Ostwald ripening are applied to each unique pair of simulated aggregates, and then changes in volume are accumulated in each hemisphere. Simulated aggregates whose volume is less than one are removed, and pairs of simulated aggregates whose edges touch are merged; the merged simulated aggregate has the combined volume of the two original simulated aggregates, and a center point that is the midpoint between the two. The e-frame aggregate movements are applied to the o-frame simulated aggregates so that movement and disappearance can be considered separately.

To determine the percent accuracy of the o-simulation, for each "o-frame::eframe" pair we find the number of pairs where the simulated aggregate matches the experimental aggregate, and then divide this number by the initial number of aggregates at the first e-frame. A pair is scored as a match if it exists or does not exist in both the e-frame and corresponding o-frame. To control for boundary conditions, the simulation is run using all of the aggregates in the frame, but the accuracy is measured only for the aggregates inside of a border 250 pixels around the edge of the image (approximately the size of two large aggregates). This reduces the possibility that aggregates just outside the image will have a significant impact on the accuracy score.

To solve for the rate of mass transfer between droplets $(\tau-1$ and $\tau-2)$ in the osimulation, we attempted every value between 0 and 1500 using increments of 10 . We determined which values for $\tau-1$ and $\tau-2$ are best for the 15 training replicates by trying every combination and selecting the pair that maximized the mean area under the accuracy plot. The decision to calculate two values for $\tau$ was made after observing a change in the collective behavior of the swarm in the middle of development. Evidence of this change appeared in the accuracy plots when a single value for $\tau$ was used. The single $\tau$ plots display an early drop in accuracy that recovers around the middle of the simulation. A single $\tau$ must represent the average rate of mass transfer for the entire o-simulation, so the drop in the accuracy plot means that the o-simulation is faster than the experiment in the beginning, and the experiment catches up by the end. These kinds of synchronous changes in behavior are not surprising for a multicellular bacterium like $M$. xanthus, and may be the result of quorum sensing or some other cell signaling system. At this point, however, we have simply observed and noted the behavior and its effect, but proposing a mechanism would be premature.

1. Mignot, T. The elusive engine in Myxococcus xanthus gliding motility. Cell. Mol. Life Sci. 64, 27332745 (2007).

2. Zhang, H. et al. Quantifying aggregation dynamics during Myxococcus xanthus development. J. Bacteriol. 193, 5164-5170 (2011).

3. Jelsbak, L. \& Søgaard-Andersen, L. Cell behavior and cell-cell communication during fruiting body morphogenesis in Myxococcus xanthus. J. Microbiol. Methods 55, 829-839 (2003).

4. Curtis, P. D., Taylor, R. G., Welch, R. D. \& Shimkets, L. J. Spatial organization of Myxococcus xanthus during fruiting body formation. J. Bacteriol. 189, 9126-9130 (2007).

5. Xie, C., Zhang, H., Shimkets, L. \& Igoshin, O. Statistical image analysis reveals features affecting fates of Myxococcus xanthus developmental aggregates. Proc. Natl. Acad. Sci. USA 108, 5915-5920 (2011).

6. Zhao, K. et al. Psl trails guide exploration and microcolony formation in Pseudomonas aeruginosa biofilms. Nature 497, 388-391 (2013).

7. Vasiev, B., Hogeweg, P. \& Panfilov, A. Simulation of Dictyostelium Discoideum via reaction diffusion model. Phys. Rev. Lett. 73, 3173 (1994).

8. Sozinova, O., Jiang, Y., Kaiser, D. \& Alber, M. A three-dimensional model of Myxobacterial aggregation by contact-mediated interactions. Proc. Natl. Acad. Sci. USA 102, 11308-11312 (2005).

9. Igoshin, O. A., Welch, R. D., Kaiser, D. \& Oster, G. Waves and aggregation patterns in Myxobacteria. Proc. Natl. Acad. Sci. USA 101, 4256-4261 (2004).

10. Igoshin, O. A., Goldbeter, A., Kaiser, D. \& Oster, G. A biochemical oscillator explains several aspects of Myxococcus xanthus behavior during development. Proc. Natl. Acad. Sci. USA 101, 15760-15765 (2004).

11. Igoshin, O. A., Kaiser, D. \& Oster, G. Breaking symmetry in Myxobacteria. Curr Biol. 14, R459-R462 (2004). 
12. Sliusarenko, O., Zusman, D. R. \& Oster, G. Aggregation during fruiting body formation in Myxococcus xanthus is driven by reducing cell movement. J. Bacteriol. 189, 611-619 (2007).

13. Rump, T. Coarsening processes in thin liquid films: Analysis and numerics. Diss. Bonn University urn:nbn:de:hbz:5N-14443 (2008).

14. Welch, R. D. \& Kaiser, D. Cell behavior in traveling wave patterns of myxobacteria. Proc. Natl. Acad. Sci. USA 98, 14907-14912 (2001).

15. Shimkets, L. J. \& Kaiser, D. Induction of coordinated movement of Myxococcus xanthus cells. J. Bacteriol. 152, 451-461 (1982).

16. Igoshin, O. A., Mogilner, A., Welch, R. D. Kaiser, D. \& Oster, G. Pattern formation and traveling waves in Myxobacteria: Theory and modelling. Proc. Natl. Acad. Sci. USA 98, 14913-14918 (2001)

17. Wolgemuth, C., Hoiczyk, E., Kaiser, D. \& Oster, G. How Myxobacteria glide. Curr. Biol. 12, 369-377 (2002).

18. Jelsbak, L. \& Søgaard-Andersen, L. Pattern formation: Fruiting body morphogenesis in Myxococcus xanthus. Curr. Opin. Microbiol. 3, 637-642 (2000).

19. Jelsbak, L. \& Søgaard-Andersen, L. Pattern formation by a cell surface-associated morphogen in Myxococcus xanthus. Curr. Opin. Microbiol. 99, 2032-2037 (2002).

20. Nan, B. et al. Myxobacteria gliding motility requires cytoskeleton rotation powered by proton motive force. Proc. Natl. Acad. Sci. USA 108, 2498-503 (2011).

21. Mauriello, E. M. F., Mignot, T., Yang, Z. \& Zusman, D. R. Gliding motility revisited: How do the Myxobacteria move without flagella? Microbiol. Mol. Biol. Rev. 74, 229-249 (2010).

22. Spormann, A. M. \& Kaiser, D. Gliding mutants of Myxococcus xanthus with high reversal frequencies and small displacements. J. Bacteriol. 181, 2593-2601 (1999).

23. Blackhart, B. D. \& Zusman, D. R. "Frizzy" genes of Myxococcus xanthus are involved in control of frequency of reversal of gliding motility. Proc. Natl. Acad. Sci. USA 82, 8767-8770 (1985).

24. Sager, B. \& Kaiser, D. Two cell-density domains within the Myxococcus xanthus fruiting body. Proc. Natl. Acad. Sci. USA 90, 3690-3694 (1993).

25. Dao, D. N., Kessin, R. H. \& Ennis, H. L. Developmental cheating and the evolutionary biology of Dictyostelium and Myxococcus. Microbiology 146, 1505-1512 (2000).

\section{Acknowledgments}

This research was made possible by a National Science Foundation Career award (MCB-0746066, Characterization of Transcriptional Activators that Regulate Emergent Behavior) to R.D.W., and National Science Foundation EFRI-MIKS award (EFRI-1106933, Deciphering and Controlling the Signal Processes in Bacterial Multicellular Systems and Bacteria-Host Interactions) to R.D.W. (co-PI). We would like to thank Joshua Shaevitz and Shashi Thutupalli (Princeton University) for help with interpreting the discretized 2D migration equations from Vasiev, B. et al. (1994) [ref. 7]. We would also like to thank Laura Welch for editing the manuscript.

\section{Author contributions}

F.B. created the time lapse microcinematography image stacks and jointly wrote the paper with P.P. and R.W.P.P. programmed the simulations and C\# annotator. S.A. was the first to suggest that $M$. xanthus development was like coarsening and performed initial proof-of-concept experiments. J.G. performed experiments for tracking individual cell movements.

\section{Additional information}

Supplementary information accompanies this paper at http://www.nature.com/ scientificreports

Competing financial interests: The authors declare no competing financial interests.

How to cite this article: Bahar, F., Pratt-Szeliga, P.C., Angus, S., Guo, J. \& Welch, R.D Describing Myxococcus xanthus Aggregation Using Ostwald Ripening Equations for Thin Liquid Films. Sci. Rep. 4, 6376; DOI:10.1038/srep06376 (2014).

This work is licensed under a Creative Commons Attribution-NonCommercialShareAlike 4.0 International License. The images or other third party material in this article are included in the article's Creative Commons license, unless indicated otherwise in the credit line; if the material is not included under the Creative Commons license, users will need to obtain permission from the license holder in order to reproduce the material. To view a copy of this license, visit http:// creativecommons.org/licenses/by-nc-sa/4.0/ 